\title{
Application Strategy of Advanced Power Electronics Technology in Smart Grid
}

\author{
Weiling $\mathrm{He}^{1}$, Chunyao Zhan², Xiaoling $\mathrm{He}^{3}$, Jianglu Yan ${ }^{4}$, Jiajian $\mathrm{Xie}^{5}$, Xing Li ${ }^{6}$ \\ ${ }^{1}$ Jiangxi Vocation College of Ahead Software, China \\ ${ }^{2}$ NanChang Rail Transit Co., Ltd. \\ ${ }^{3}$ Shanghai FGbioko Automobile Technology co.,Ltd \\ ${ }^{4,5,6}$ East China University of Technology
}

Keywords: Power electronics technology; Smart grid; The application strategy

\begin{abstract}
With the rapid development of China's modernization, electric power energy has an important position in China's energy which provides energy security for the continuous rapid development of China's economy. With the continuous improvement of people's living standards, the application of electric power and electronic technology in smart grid can effectively guarantee the electricity demand of people's daily life. Under the circumstances of economic globalization, the smart power grid has become the common direction of global power grid development. However, as an important means of smart grid operation, advanced power electronics technology is not only required by grid security, but also by the development of the power market. This paper analyzes the development status in China's power grid in detail, summarizing relevant experience, and analyzing the application strategy of power electronics technology in smart power grid.
\end{abstract}

With the development of China's economy and the continuous progress of science and technology, various industries have come to a crucial stage of reform and upgrading. China's power grid system also began to develop in a new direction-smart power grid. Due to the excessive development and utilization of human beings on the earth's resources, the world has entered the stage of energy crisis, a smart grid technology can effectively improve the utilization rate of resources, it can make the conform to the actual demand of electricity regulation through a collection of integration of the whole grid power usage. What's more, the smart grid technology can balance the allocation and transportation of power, providing good technical support for the effective utilization of resources in China and promoting the sustainable development of China.

\section{Advantages and characteristics of power electronics technology in smart grid}

\subsection{Significantly improve the safety and stability of power grid}

The previous role of the grid is guaranteed by the security and stability of the grid itself. Since its birth and development, the safety system and the stability of the grid itself are important development directions in any period, and the safety of smart grid is the primary issue. The development of modern power grid is becoming more and more automatic and intelligent, and the safety and the stability of power grid have been greatly improved. Smart power grid is equipped with advanced sensing technology and measurement technology, which can timely record collect the changes when the power grid itself changes, so as to facilitate the grid staff to manage and maintain the entire power grid and largely avoid the occurrence of dangerous accidents. And smart grid can withstand evil imperial foreign attack, in the years when technology develops rapidly, the grid system is also inevitably become the target of criminals, and the smart grid ability has the characteristics of self-healing and against attacks, it can carry on the key protection independent parts of key information, fully ensure the power grid in intelligence as well as its own security and job stability. 


\subsection{Improve grid efficiency to reduce pollutant emissions}

Smart grid has advanced control method, equipment technology and advanced intelligent decision system. However, advanced control methods and equipment technologies can effectively reduce the failure of each component of the grid in the actual use process and improve the quality of power transmission. Advanced and sophisticated scientific and technological means can quickly diagnose the problems in the presence of power network problems and obtain appropriate and rigorous solutions through intelligent decision-making system. At the same time, smart grid has strong compatibility and can integrate centralized power plants and decentralized power resources. It can be compatible with centralized access to other distributed power resources, so as to better manage and regulate power resource in hierarchical manner and improve grid efficiency. The characteristics of smart power grid can effectively promote the development of power grid. At the same time, power energy is also green energy. The development of power grid will promote the popularization of green energy, so as to stick to the emission of pollutants and promote the sustainable development of China's.

\section{The construction of China's modern smart power grid}

Compared with foreign countries, China's smart grid construction started late, and the new concept of smart grid makes China's power grid development lag behind the developed countries. Relevant technical experience is insufficient, and there is no successful case for reference in China, which leads to the slow development of smart automation of power grid in China. What about China's smart grid?

\subsection{The lack of electricity and the phenomenon of nest electricity coexist, the construction of interregional network lags behind}

China's primary energy distribution and the imbalance of regional economic development determine the inevitability of large-scale trans-regional allocation of resources and optimized allocation nationwide. With the rapid development of China's economy and the sustained and rapid growth of power demand, the imbalance between the local power development mode and the distribution of resources and productivity has become increasingly prominent. The lack of electricity and the phenomenon of dimples co-exist, the construction of interregional networking lags behind, the transmission and exchange capacity between regions is insufficient, the distribution range and efficiency of power resources are greatly limited, and the ability to optimize the allocation of resources in a wider range needs to be improved. In addition, due to the increasingly prominent environmental pressure caused by frequent haze weather in eastern regions, it is also required to speed up the construction system centering on electricity, so as to realize electricity comes from afar"

\subsection{Insufficient access and control capacity of new energy}

New energy sources such as wind power and photovoltaic power are developing rapidly in China. On the one hand, the construction of wind power base is accelerating, showing the characteristics of large-scale and intensive development. On the other hand, distributed new energy and other forms of power generation are booming, and there is the possibility of explosive growth in the future. This poses a major challenge to the operation of the grid.

\subsection{The application scale, scope and depth of smart grid technology are relatively low}

Since 2009, the state grid corporation of China has applied a number of advanced applicable technologies, such as state monitoring of transmission and transformation equipment, fault comprehensive analysis alarm and distribution network self-healing. However, overall, the scale, scope and depth of these technologies are still relatively low, which requires further promotion. At the same time, more attention should be paid to the application of advanced network information and automatic control and other basic technologies to have a further improvement in intelligent level of online intelligent analysis, early warning, decision-making, control and other aspects of the 
grid, as well as to meet the requirements of coordinated control of all levels of the grid, and support the integrated operation of the smart grid.

\subsection{Intelligent interaction with customers needs to be improved}

With the rapid development of the distributed power supply on the user side and the distribution side, especially with the large-scale use of rooftop solar power generation and electric vehicles, the two-way interaction of power flow and information flow in the grid are constantly strengthened, which will have a significant impact on the operation and management of the grid. The users can through the electronic terminal and power grid company formed connection between network interaction and instant, realizing real-time, high-speed, bidirectional power data and read from the overall effect, electric power, telecommunications, television, intelligent home appliances control and battery charge integration of multipurpose development, realizing all kinds of power supply and customer terminal seamless interconnection to the grid, optimizing the grid management, forming a grid brand-new service function, improving the reliability of the power grid, usability, and overall efficiency.

\section{Application strategy of power electronics technology in smart grid}

\subsection{High-voltage direct current transmission technology}

High voltage direct current transmission (HVDC) is a long distance power transmission using stable direct current. This technology has obvious advantages compared with traditional transmission mode in long-distance transmission of power. There is no synchronization problem for HVDC, and its own cost is very low, it requires few lines and reduces the capital needs for line construction. Because direct current usually takes one or two lines for power transmission, the loss of power in long-distance power transmission is relatively small. HVDC technology is quite mature in submarine cable transmission. Compared with ac transmission, dc transmission technology can withstand higher power, and will not generate magnetic induction on the line shell loss. The application of power electronics technology can better establish HVDC transmission technology and scientific transmission mode.

\subsection{Flexible ac transmission technology}

Flexible ac transmission technology is a kind of comprehensive power electronics technology, which has many characteristics. Flexible ac transmission technology can optimize ac transmission technology and improve the flexibility of ac power grid. At the same time, flexible ac transmission technology is flexible in operation, which can effectively adjust the distribution of power grid and improve the overall stability of power grid. The flexible ac transmission technology also has the function of improving the transmission capacity, less transmission effect caused by line fault and reducing the loss caused by line safety accident. As China's intelligent power grid is still in the initial stage of development, flexible ac transmission technology can reduce energy consumption, so it is necessary to fully develop this technology. The construction of smart grid should take full account of the interconnection of different lines, give play to the synergistic effect of flexible ac transmission technology, and accelerate the construction of smart grid according to the actual situation.

\subsection{High-voltage frequency conversion technology}

High voltage frequency conversion technology is the most common technology in power system, and it is also a power technology that people can often contact in life. This technology is to reduce energy consumption and improve energy efficiency through transformers. With the continuous development of electronic technology, this technology has been applied for many years, and its development degree has been quite mature. It has been widely used in large petrochemical industry, metal smelting industry and other industries. The practical application of high voltage frequency conversion technology in power transmission has proved its important position and positive role. While improving the efficiency of electric energy utilization, it also reduces the pollution to the 
environment. The establishment of smart grid is inseparable from mature high-voltage frequency conversion technology. Power electronics technology can improve the application effect of high-voltage frequency conversion technology, and actually improve the application effect.

\subsection{Intelligent switch technology}

A key technology in the development of smart grid is intelligent switch technology. The switch is distributed in each component structure of the power grid. The actual control of the current and voltage of the circuit protects the normal operation of the circuit to determine the power supply and cut off. Intelligent switch plays a key role in the intelligent power grid. The wide application of intelligent switch can effectively guarantee the stability of the circuit and avoid the circuit problems caused by the current derangement and line overload. Compared with the traditional switch, the intelligent switch can make decisions by self-perception of the actual situation of the circuit, and at the same time, it can realize remote control by connecting with the computer, which greatly improves the control strength of the staff on the circuit. Be able to cut off the power supply in time when the problem accident occurs, so as to avoid the accident developing towards worse direction. The use of intelligent switch ensures the safety of both ends of the transmission equipment and the power users' equipment. In case of unstable current and unstable voltage, the power supply is cut off in time to avoid damage of the power equipment. It also protects the safety of the transmission line. Under the premise of continuous improvement of science and technology, the advantages of intelligent switch are increasing, which greatly promotes the process of intelligent development of China's power grid.

\section{Conclusion}

With China's reform and opening up and economic globalization, all industries in China should be in line with international standards. Grid intellectualization has been the development direction of international power grid. The application of modern power electronics technology to the development of grid intelligence can effectively improve the safety and stability of the grid and the process of grid intelligence. Relevant staff should recognize the gap between actual demand and development level, flexibly apply power electronics technology and relevant high-tech technology to fully promote the intelligent development of China's power grid. In the construction of intelligent power grid, we should use high and new technology actively and give full play to its positive role to drive the progress of the industry and promote China's economic development.

\section{References}

[1] Wenliang Zhang, Xiang Liu, Mingjun Wang, Xusheng Yang. Research progress and development trend of smart grid [J]. Grid technology,2009(13).

[2] Huan Yang. Analysis of the application of advanced power electronics technology in smart grid [J]. Science Chinese,2015(14).

[3] Li Zhang. Application research of advanced power electronics technology in smart grid [J]. Journal of education college of Jilin province (middle),2015(09).

[4] Shibiao Huang. Application of advanced power electronics technology in smart grid [J]. Electronic world,2016(04).

[5] Yuye Han. Application of advanced power electronics technology in smart grid [J]. Hebei enterprise,2016(11). 